\title{
Global Burden and Attributable Risk Factors of Acute Lymphoblastic Leukemia in 204 Countries and Territories from 1990-2019: Estimation Based on Global Burden of Disease Study 2019
}

\section{Yuefen Hu}

Shandong University Cheeloo College of Medicine

\section{Xiuping Zhang}

Jinan Central Hospital Affiliated to Shandong University

Aijun Zhang

Shandong University Qilu Hospital

Yu Hou

Shandong University Qilu Hospital

Yang Liu

Shandong University Qilu Hospital

Qizhao Li

Shandong University Cheeloo College of Medicine

\section{Yawen Wang}

Shandong University Qilu Hospital

\section{Yafei Yu}

Shandong University Cheeloo College of Medicine

\section{Ming Hou}

Shandong University Qilu Hospital

Jun Peng

Shandong University Qilu Hospital

\section{Xiaorong Yang}

Shandong University Qilu Hospital

Shuqian Xu ( $\square$ shuqian.xu@email.sdu.edu.cn )

Shandong University Qilu Hospital https://orcid.org/0000-0001-7071-8550

\section{Research Article}

Keywords: Acute lymphoblastic leukemia, Global burden, Temporal trend, Risk factors, Cancer statistic

Posted Date: May 18th, 2021 
DOI: https://doi.org/10.21203/rs.3.rs-508326/v1

License: (c) (1) This work is licensed under a Creative Commons Attribution 4.0 International License. Read Full License

Version of Record: A version of this preprint was published at Hematological Oncology on October 24th, 2021. See the published version at https://doi.org/10.1002/hon.2936. 


\section{Abstract}

Purpose: To provide a foundational guideline for policy-makers to efficiently allocate medical resources in the context of population aging and growth, a latest spatial distribution and temporal trend of acute lymphoblastic leukemia (ALL) along with attributable risk factors by sex and age were mapped.

Methods: Based on the Global Burden of Disease Study 2019, we calculated the estimated annual percentage change (EAPC) values to quantify temporal trends in morbidity and mortality of ALL. We used applied Spearman rank correlation to estimate the relationship between the EAPC and potential influence factors. The population attributable fraction of potential risk factors for ALL-related disability-adjusted life years (DALYs) were estimated by the comparative risk assessment framework.

Results: We found that new ALL cases increased significantly by $129 \%$ worldwide, and the agestandardized incidence rate (ASIR) increased by 1.61 percent per year. The proportion of elder patients sharply increased, especially within the higher socio-demographic index (SDI) region. Smoking and high body mass index remained the predominant risk factors for ALL-related mortality. Notably, the contribution of high body mass index presented an increasing trend.

Conclusion: The global burden of ALL has steadily increased, especially in middle SDI region. Health measures should be taken into consideration to improve the treatment of elders with ALL due to a great proportion in the higher SDI region. Attention should be paid to the environmental problems caused by industrial development in low SDI areas.

\section{Introduction}

Leukemias are a group of malignant clonal disorders that originate in hematopoietic stem cells and are characterized by an increased number of leucocytes in the blood and/or the bone marrow (Juliusson and Hough 2016). Acute lymphoblastic leukemia (ALL) is a common subtype of leukemia presented with chromosomal abnormalities and genetic alterations involved in differentiation and proliferation of lymphoid precursor cells (Florent Malard 2020). The reported incidence of ALL is 1.7 cases per 100000 individuals per year within the USA, with approximately 6150 new cases estimated in 2020, exceeding the 5970 new cases in 2017. In the world, new cases had reached 153 320, with age-standardized morbidity reaching $1.96 / 100000$ by 2019 . It is frequently diagnosed in children and young adults, with incidence peaks between 1 and 4 years of age. Notably, the incidence of ALL in the elderly is also increasing each year (Florent Malard 2020; Dong et al. 2020; 'National Cancer Institute. Cancer Stat Facts: Leukemia - Acute Lymphocytic Leukemia (ALL)').

Although the mechanisms of ALL are not completely understood, several factors are thought to be involved, such as ionizing radiation (a causal exposure was established in childhood ALL), infection (the first indicated and the strongest candidate for the cause of ALL) and inherited susceptibility. At present, the first-line treatment of ALL typically comprises 3 phases: remission-induction, intensification (or consolidation), and continuation (or maintenance) (Inaba, Greaves, and Mullighan 2013; Terwilliger and 
Abdul-Hay 2017; Network). More than $80 \%$ of children between 1 and 18 years of age are expected to relieve effectively (Vrooman and Silverman 2016; Pui et al. 2009; Veerman et al. 2009), but only 30\% to $40 \%$ of first salvage and $10 \%$ to $20 \%$ of second salvage regimens achieve complete remission (Gregory 2019; Ronson, Tvito, and Rowe 2016). Adult patients relapse rate was still high (Linker et al. 1987), and $20 \%$ of children experienced a relapse (Vrooman and Silverman 2016). Hence, the heterogeneity of the ALL-related burden internationally is profound. Analysis of the global burden of ALL by region, sex and age will provide important guidance for its biology, prevention and treatment.

The Global Burden of Diseases (GBD) Study 2019 comprehensively collected an extensive set of data sources and applied novel statistical methods to estimate the incidence, mortality and disability-adjusted life years (DALYs) caused by various diseases along with corresponding risk factors in 204 countries and territories from 1990 to 2019 (Pourshams et al. 2019). Given the disease burden of ALL is unclear across geographies, we conducted this study to investigate the number of incidence cases, deaths, DALYs, and corresponding age-standardized rates of ALL. Moreover, we presented the changes in ALL-related DALYs attributed to potential risk factors. Our research can inform policy-makers on how to allocate limited public health resources reasonably and effectively.

\section{Methods}

\section{Study data}

The GBD 2019 study estimates 369 diseases burden along with 87 risk factors in 204 countries and territories by gender, age group, and location. The detailed methods for the statistical estimation could be found on the supporting website, http://ghdx.healthdata.org/gbd-2019/code (Collaborators 2020a; Collaborators 2020b). In brief, the cancer burden estimation in the GBD study was originated from various national cancer registries and aggregate cancer database registries, such as Cancer Incidence in Five Continents (CI5), Nordic Cancer Registries database (NORDCAN), Surveillance, Epidemiology, and End Results (SEER). All ICD-9 and ICD-10 codes pertaining to ALL (204.0-204.02 and C91.0-C91.02, C91.2C91.32, C91.6-C91.62, respectively) were included in these estimates. The 95\% uncertainty intervals (UIs) for all estimates in the GBD Study were calculated based on the 25th and 975th ordered values of 1,000 randomly selected posterior distributions. All geographical regions around the world were categorized into 21 GBD regions (e.g. High income North America) and seven super GBD regions (e.g. High income). Based on socio-demographic index (SDI) distribution, countries and territories were further divided into five SDI regions, including high, high-middle, middle, low-middle, and low SDI regions. The SDI is a comprehensive indicator reflecting the level of health development, which integrates per capita income, education level, and fertility rate. We retrieved annual numbers and rates of incidence cases, deaths, and disability-adjusted life years (DALYs) of ALL by sex, location, and age group (5-year groups within the ages of 0 to 94 years, and $\geq 95$ years) from the Global Health Data Exchange (GHDx) website, http://ghdx.healthdata.org/gbd-results-tool. Age-standardized rates (ASRs) were estimated according to the WHO 2001 world population distribution. We further downloaded the annual SDI information of each region for correlation analysis. According to the GBD database, four well-judged risk factors contributed 
to ALL burden, including smoking, high body mass index, occupational exposure to benzene, and occupational exposure to formaldehyde. The attributable burden of potential risk factors in the GBD Study was calculated based on the comparative risk assessment framework with six steps: identifying cogent risk-outcome pairs, estimating relative risk using systematic review, collecting the spatial exposure levels, determining the theoretical minimum level of exposure, calculating the population attributable fractions, and assessing combined attributable proportion by considering the mediating effect (Collaborators 2020b).

\section{Statistical analysis}

To avoid interference from changes in age distribution and population sample size, we used the ASR to estimate the difference in the burden of ALL by year, sex, and region. The annual change trend of the burden of ALL was quantified using the estimated annual percentage change (EAPC), which was

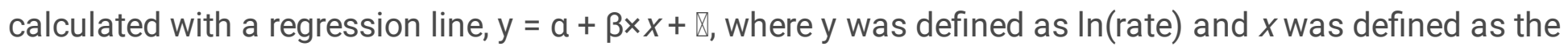
calendar year (Yang et al. 2021). The EAPC was calculated with the formula, $100 \times(\exp (\beta)-1)$. Considering the influence of the standard error of the fitted model for EAPC, the $95 \%$ confidence interval $(95 \% \mathrm{Cl})$ of EAPC should be further expressed in our results. Due to non-normal distribution, we calculated the Spearman rank correlation coefficient to estimate the relationship between the EAPC and SDI value at the national level. All statistical analyses and char presenting were performed using the open-source $\mathrm{R}$

program version 4.0.3 (https://www.R-project.org/), with ggplot2, corrplot, RColorBrewer, and other related packages. A two-sided $P$ value less than 0.05 was considered statistically significant.

\section{Results}

\section{Incidence tendency and variation in ALL}

Globally, new cases of ALL increased from 66810 in 1990 to 153320 in 2019, and the ASIR increased by 1.61 per year (95\% Cl 1.52 1.71) during this period (Table 1). Males had more opportunities to suffer from ALL than females. And the male-to-female ratio was 1.24 in 1990, then 1.22 in 2019 (Additional file 1: Fig. S1A). Although the ASIR of both sexes continued to climb, males' ASIR increased more quickly than females' in most age groups (Table 1, Additional file 1: Fig. S2A).

When we compared SDI regions, the middle SDI region had the highest incidence in 1990 (17 450 cases), while the high-middle SDI region had the highest in 2019 (52 070 cases), with the most rapid growth of ASIR (ASIR: 1.51 in 1990 and 3.39 in 2019, EAPC = 3.08, 95\% Cl 2.79 3.37; Table 1, Additional file 1: Fig. S3A). High SDI region always had the highest ASIR, and had a significant upward trend from 1990 to 2019 (ASIR: 1.02 in 1990 and 3.52 in 2019, EAPC = 1.72, 95\% Cl 1.44 2). To our delight, the ASIR of ALL decreased in low-middle and low SDI regions, the latter had the lowest incidence burden (Additional file 1: Fig. S1A, Table 1). 


\section{Table 1}

Incidence and age-standardized incidence rate per 100000 people for ALL in 1990 and 2019.

\begin{tabular}{|c|c|c|c|c|c|}
\hline & 1990 & & 2019 & & 1990-2019 \\
\hline \multirow[t]{2}{*}{ Characteristics } & $\begin{array}{l}\text { Incident } \\
\text { cases }\end{array}$ & $\begin{array}{l}\text { ASIR per } \\
100000\end{array}$ & \multirow{2}{*}{$\begin{array}{l}\text { Incident cases } \\
\text { No. } \times 10^{3}(95 \% \\
\text { UI) }\end{array}$} & $\begin{array}{l}\text { ASIR per } \\
100000\end{array}$ & $\begin{array}{l}\text { EAPC in } \\
\text { ASIR }\end{array}$ \\
\hline & $\begin{array}{l}\text { No. } \times 10^{3} \\
(95 \% \text { UI })\end{array}$ & $\begin{array}{l}\text { No. }(95 \% \\
\text { UI) }\end{array}$ & & $\begin{array}{l}\text { No. }(95 \% \\
\text { UI) }\end{array}$ & No. $(95 \% \mathrm{Cl})$ \\
\hline Overall & $\begin{array}{l}66.81(52.91 \\
, 88.25)\end{array}$ & $\begin{array}{l}1.23(0.99 \\
, 1.58)\end{array}$ & $\begin{array}{l}153.32(128.58 \\
, 170.55)\end{array}$ & $\begin{array}{l}1.96(1.64 \\
, 2.17)\end{array}$ & $\begin{array}{l}1.61(1.52 \\
, 1.71)\end{array}$ \\
\hline \multicolumn{6}{|l|}{ Sex } \\
\hline Males & $\begin{array}{l}36.94(28.12 \\
, 50.27)\end{array}$ & $\begin{array}{l}1.36(1.06 \\
, 1.79)\end{array}$ & $\begin{array}{l}84.12(66.44 \\
, 96.18)\end{array}$ & $\begin{array}{l}2.18(1.73 \\
, 2.49)\end{array}$ & $\begin{array}{l}1.71(1.62 \\
, 1.81)\end{array}$ \\
\hline Females & $\begin{array}{l}29.86(22.64 \\
, 51.3)\end{array}$ & $\begin{array}{l}1.11(0.85 \\
, 1.84)\end{array}$ & $\begin{array}{l}69.19(52.85 \\
, 82.35)\end{array}$ & $\begin{array}{l}1.75(1.34 \\
, 2.08)\end{array}$ & $\begin{array}{l}1.49(1.38 \\
, 1.6)\end{array}$ \\
\hline \multicolumn{6}{|l|}{ SDI region } \\
\hline High SDI & $\begin{array}{l}16.41(13.54 \\
, 17.69)\end{array}$ & $\begin{array}{l}2.03(1.69 \\
, 2.19)\end{array}$ & $\begin{array}{l}40.1(34.89 \\
, 45.77)\end{array}$ & $\begin{array}{l}3.52(3.08 \\
, 4.05)\end{array}$ & $\begin{array}{l}1.72(1.44 \\
, 2)\end{array}$ \\
\hline High-middle SDI & $\begin{array}{l}16.88(14.91 \\
, 19.38)\end{array}$ & $\begin{array}{l}1.51(1.33 \\
, 1.74)\end{array}$ & $\begin{array}{l}52.07(43.22 \\
, 59.67)\end{array}$ & $\begin{array}{l}3.39(2.8 \\
, 3.89)\end{array}$ & $\begin{array}{l}3.08(2.79 \\
, 3.37)\end{array}$ \\
\hline Middle SDI & $\begin{array}{l}17.45(13.04 \\
, 24.02)\end{array}$ & $\begin{array}{l}0.96(0.74 \\
, 1.31)\end{array}$ & $\begin{array}{l}37.89(27.19 \\
, 43.65)\end{array}$ & $\begin{array}{l}1.6(1.16 \\
, 1.83)\end{array}$ & $\begin{array}{l}2.11(1.71 \\
, 2.52)\end{array}$ \\
\hline Low-middle SDI & $\begin{array}{l}10.23(6.64 \\
, 17.14)\end{array}$ & $\begin{array}{l}0.76(0.52 \\
, 1.2)\end{array}$ & $\begin{array}{l}11.66(9.86 \\
, 13.98)\end{array}$ & $\begin{array}{l}0.66(0.56 \\
, 0.8)\end{array}$ & $\begin{array}{l}-0.45(-0.5 \\
-0.41)\end{array}$ \\
\hline Low SDI & $\begin{array}{l}5.8(2.83 \\
, 13.44)\end{array}$ & $\begin{array}{l}0.78(0.44 \\
, 1.61)\end{array}$ & $\begin{array}{l}8.03(5.9 \\
, 10.29)\end{array}$ & $\begin{array}{l}0.59(0.45 \\
, 0.73)\end{array}$ & $\begin{array}{l}-0.85(-0.92 \\
-0.78)\end{array}$ \\
\hline
\end{tabular}

GBD region

\begin{tabular}{|c|c|c|c|c|c|}
\hline $\begin{array}{l}\text { High-income Asia } \\
\text { Pacific }\end{array}$ & $\begin{array}{l}4.31(3.47 \\
, 5.02)\end{array}$ & $\begin{array}{l}2.47(2.03, \\
2.88)\end{array}$ & $\begin{array}{l}11.85(9.57 \\
, 13.75)\end{array}$ & $\begin{array}{l}5.42(4.56, \\
6.33)\end{array}$ & $\begin{array}{l}2.81 \\
(2.52,3.1)\end{array}$ \\
\hline $\begin{array}{l}\text { High-income North } \\
\text { America }\end{array}$ & $\begin{array}{l}3.35(2.81 \\
, 3.58)\end{array}$ & $\begin{array}{l}\text { 1.21 (1.02, } \\
1.31)\end{array}$ & $5.3(4.44,6.3)$ & $\begin{array}{l}1.32(1.11, \\
1.57)\end{array}$ & $\begin{array}{l}0.3 \\
(0.19,0.41)\end{array}$ \\
\hline Western Europe & $\begin{array}{l}11.75(9.61 \\
, 12.83)\end{array}$ & $\begin{array}{l}3.18(2.61 \\
3.51)\end{array}$ & $\begin{array}{l}29.59(24.84 \\
, 35.04)\end{array}$ & $\begin{array}{l}6.27(5.29 \\
7.42)\end{array}$ & $\begin{array}{l}1.95 \\
(1.47,2.44)\end{array}$ \\
\hline Australasia & $\begin{array}{l}0.37(0.3 \\
, 0.42)\end{array}$ & $\begin{array}{l}1.85(1.49, \\
2.1)\end{array}$ & $\begin{array}{l}0.85(0.65 \\
, 1.12)\end{array}$ & $\begin{array}{l}2.46(1.89, \\
3.24)\end{array}$ & $\begin{array}{l}0.55 \\
(0.29,0.82)\end{array}$ \\
\hline $\begin{array}{l}\text { Tropical Latin } \\
\text { America }\end{array}$ & $\begin{array}{l}1.9(1.72 \\
, 2.19)\end{array}$ & $\begin{array}{l}1.13(1.02, \\
1.3)\end{array}$ & $\begin{array}{l}2.18(1.86 \\
, 2.43)\end{array}$ & $\begin{array}{l}1.06(0.91, \\
1.2)\end{array}$ & $\begin{array}{l}0.04 \\
(-0.11,0.19)\end{array}$ \\
\hline Andean Latin & $0.64(0.5$ & 1.47 (1.19, & $1.12(0.76$ & 1.78 (1.21, & 0.83 \\
\hline
\end{tabular}




\begin{tabular}{|c|c|c|c|c|c|}
\hline America & $, 0.85)$ & $1.95)$ & ,1.44) & 2.29) & $(0.71,0.96)$ \\
\hline $\begin{array}{l}\text { Central Latin } \\
\text { America }\end{array}$ & $\begin{array}{l}3.1(2.85 \\
, 3.49)\end{array}$ & $\begin{array}{l}1.66(1.55 \\
1.91)\end{array}$ & $\begin{array}{l}5.65(4.47 \\
, 6.67)\end{array}$ & $\begin{array}{l}2.3(1.81, \\
2.71)\end{array}$ & $\begin{array}{l}1.35 \\
(1.25,1.45)\end{array}$ \\
\hline $\begin{array}{l}\text { Southern Latin } \\
\text { America }\end{array}$ & $\begin{array}{l}0.65(0.6 \\
, 0.73)\end{array}$ & $\begin{array}{l}1.29(1.19 \\
1.43)\end{array}$ & $\begin{array}{l}1.15(0.88 \\
, 1.46)\end{array}$ & $\begin{array}{l}1.72(1.32, \\
2.17)\end{array}$ & $\begin{array}{l}1.14 \\
(0.99,1.28)\end{array}$ \\
\hline Caribbean & $\begin{array}{l}0.44(0.33 \\
, 0.64)\end{array}$ & $\begin{array}{l}1.17(0.88 \\
1.67)\end{array}$ & $0.6(0.45,0.77)$ & $\begin{array}{l}1.31(0.98, \\
1.71)\end{array}$ & $\begin{array}{l}0.53 \\
(0.41,0.65)\end{array}$ \\
\hline Eastern Europe & $\begin{array}{l}4.59(4.21 \\
, 5.2)\end{array}$ & $\begin{array}{l}2.13(1.93 \\
2.41)\end{array}$ & $5.4(4.73,6.15)$ & $\begin{array}{l}2.28(2 \\
2.58)\end{array}$ & $\begin{array}{l}0.17 \\
(-0.35,0.7)\end{array}$ \\
\hline Central Europe & $\begin{array}{l}1.55(1.32 \\
, 1.7)\end{array}$ & $\begin{array}{l}1.28(1.11 \\
1.41)\end{array}$ & $2.93(2.48,3.5)$ & $\begin{array}{l}2.15(1.83 \\
2.55)\end{array}$ & $\begin{array}{l}1.97 \\
(1.84,2.11)\end{array}$ \\
\hline Central Asia & $\begin{array}{l}0.97(0.8 \\
, 1.18)\end{array}$ & $\begin{array}{l}1.31(1.1 \\
1.55)\end{array}$ & $\begin{array}{l}0.94(0.78 \\
, 1.13)\end{array}$ & $\begin{array}{l}1.03(0.86 \\
1.23)\end{array}$ & $\begin{array}{l}-0.96 \\
(-1.07,-0.85)\end{array}$ \\
\hline $\begin{array}{l}\text { North Africa and } \\
\text { Middle East }\end{array}$ & $\begin{array}{l}4.19(2.56 \\
, 6.39)\end{array}$ & $\begin{array}{l}1.1(0.71 \\
1.61)\end{array}$ & $\begin{array}{l}6.18(4.31 \\
, 7.53)\end{array}$ & $\begin{array}{l}1.08(0.76, \\
1.3)\end{array}$ & $\begin{array}{l}-0.17 \\
(-0.31,-0.02)\end{array}$ \\
\hline South Asia & $\begin{array}{l}9.13(5.64 \\
, 16.27)\end{array}$ & $\begin{array}{l}0.69 \\
1.17)\end{array}$ & $\begin{array}{l}9.3(7.66 \\
, 11.39)\end{array}$ & $\begin{array}{l}0.51(0.42, \\
0.63)\end{array}$ & $\begin{array}{l}-1.29 \\
(-1.39,-1.19)\end{array}$ \\
\hline Southeast Asia & $\begin{array}{l}4.38(2.83 \\
, 7.09)\end{array}$ & $\begin{array}{l}0.86(0.59 \\
1.37)\end{array}$ & $\begin{array}{l}5.21(4.27 \\
, 6.54)\end{array}$ & $\begin{array}{l}0.82(0.67 \\
1.02)\end{array}$ & $\begin{array}{l}-0.14 \\
(-0.18,-0.11)\end{array}$ \\
\hline East Asia & $\begin{array}{l}10.89(7.67 \\
, 17.42)\end{array}$ & $\begin{array}{l}0.91(0.65 \\
1.47)\end{array}$ & $\begin{array}{l}58.11(39.98 \\
, 69.92)\end{array}$ & $\begin{array}{l}3.73(2.52, \\
4.47)\end{array}$ & $\begin{array}{l}5.86 \\
(4.87,6.86)\end{array}$ \\
\hline Oceania & $\begin{array}{l}0.04(0.02 \\
, 0.06)\end{array}$ & $\begin{array}{l}0.53(0.34 \\
0.82)\end{array}$ & $\begin{array}{l}0.07(0.04 \\
, 0.11)\end{array}$ & $\begin{array}{l}0.5(0.33 \\
0.76)\end{array}$ & $\begin{array}{l}-0.34 \\
(-0.48,-0.2)\end{array}$ \\
\hline $\begin{array}{l}\text { Western Sub- } \\
\text { Saharan Africa }\end{array}$ & $\begin{array}{l}1.01(0.64 \\
, 1.53)^{-}\end{array}$ & $\begin{array}{l}0.4(0.28 \\
0.56)\end{array}$ & $2.04(1.5,2.7)$ & $\begin{array}{l}0.37(0.28, \\
0.47)\end{array}$ & $\begin{array}{l}-0.33 \\
(-0.42,-0.24)\end{array}$ \\
\hline $\begin{array}{l}\text { Eastern Sub- } \\
\text { Saharan Africa }\end{array}$ & $\begin{array}{l}3.09(1.26 \\
, 8.38)\end{array}$ & $\begin{array}{l}1.05(0.51 \\
2.54)\end{array}$ & $\begin{array}{l}4.15(2.47 \\
, 5.87)\end{array}$ & $\begin{array}{l}0.79(0.51 \\
1.06)\end{array}$ & $\begin{array}{l}-0.89 \\
(-1.01,-0.76)\end{array}$ \\
\hline $\begin{array}{l}\text { Central Sub- } \\
\text { Saharan Africa }\end{array}$ & $\begin{array}{l}0.3(0.12 \\
, 0.67)\end{array}$ & $\begin{array}{l}0.39(0.18 \\
0.78)\end{array}$ & $0.5(0.32,0.78)$ & $\begin{array}{l}0.31(0.21 \\
0.48)\end{array}$ & $\begin{array}{l}-0.54 \\
(-0.67,-0.42)\end{array}$ \\
\hline $\begin{array}{l}\text { Southern Sub- } \\
\text { Saharan Africa }\end{array}$ & $\begin{array}{l}0.15(0.11 \\
, 0.2)\end{array}$ & $\begin{array}{l}0.25(0.2 \\
0.33)\end{array}$ & $0.2(0.15,0.25)$ & $\begin{array}{l}0.25(0.19 \\
0.31)\end{array}$ & $\begin{array}{l}-0.01 \\
(-0.08,0.07)\end{array}$ \\
\hline
\end{tabular}

For GBD regions, East Asia had the highest incidence in 2019, followed by Western Europe, with 58110 and 29590 cases, respectively. At the same time, East Asia had the greatest increase in ASIR (EAPC: 5.86, 95\% Cl: 4.87 6.86), followed by High-income Asia Pacific and Central Europe (Table 1). Notably, South Asia and Central Asia had the most rapid decrease in ASIR of ALL. 
At the country or territory level, San Marino had the highest ASIR in 2019 (23.28), 122 times greater than the lowest ASIR (0.19 in Namibia). The ASIR of ALL in four other countries, Italy, Andorra, Monaco, and Spain, was more than 7.0 (Fig. 1A, Additional file 2: Table S2). Further, Taiwan (Province of China) had the fastest growth in ASIR (EAPC $=8.19,95 \% \mathrm{Cl} 7.52 \sim 8.88)$, and Guatemala, South Korea, and Cyprus also presented an obvious increase in ASIR, with all EAPCs above 7.0 (Fig. 1C, Additional file 2: Table S3).

\section{Death tendency and variation in ALL}

In general, ALL-related deaths increased slightly from 41240 in 1990 to 47650 in 2019, while the ASDR of ALL decreased slightly by 0.57 per year from 0.76 in 1990 to 0.63 in 2019 (Table 2). The number of males died from ALL was 23950 in 1990 and 28170 in 2019, higher than females (17 300 in 1990 and 19480 in 2019). We found the ASDR in both sexes has been decreased, at an annual trend of $0.78 /$ year in females and 0.43/year in males (Table 2, Additional file 1: Fig. S1B). 


\section{Table 2}

Deaths and age-standardized death rate per 100000 people for ALL in 1990 and 2019.

\begin{tabular}{|c|c|c|c|c|c|}
\hline & 1990 & & 2019 & & 1990-2019 \\
\hline \multirow[t]{2}{*}{ Characteristics } & Deaths cases & $\begin{array}{l}\text { ASDR per } \\
100000\end{array}$ & Deaths cases & $\begin{array}{l}\text { ASDR per } \\
100000\end{array}$ & $\begin{array}{l}\text { EAPC in } \\
\text { ASDR }\end{array}$ \\
\hline & $\begin{array}{l}\text { No. } \times 10^{3} \\
(95 \% \text { UI })\end{array}$ & No. $(95 \% \mathrm{UI})$ & $\begin{array}{l}\text { No. } \times 10^{3}(95 \% \\
\text { UI) }\end{array}$ & No. $(95 \% \mathrm{UI})$ & No. $(95 \% \mathrm{Cl})$ \\
\hline Overall & $\begin{array}{l}41.24(32.02 \\
, 56.59)\end{array}$ & $\begin{array}{l}0.76(0.61 \\
, 1.02)\end{array}$ & $\begin{array}{l}47.65(39.37 \\
, 53)\end{array}$ & $\begin{array}{l}0.63(0.52 \\
, 0.7)\end{array}$ & $\begin{array}{l}-0.57(-0.65 \\
,-0.49)\end{array}$ \\
\hline
\end{tabular}

Sex

$\begin{array}{llllll}\text { Males } & 23.95(17.66 & 0.89(0.68 & 28.17(22.22 & 0.75(0.59 & -0.43(-0.53 \\ & , 33.52) & , 1.2) & , 31.87) & , 0.85) & ,-0.33) \\ \text { Females } & 17.3(12.07 & 0.64(0.46 & 19.48(14.98 & 0.5(0.39 & -0.78(-0.84 \\ & , 33.43) & , 1.2) & , 23.25) & , 0.61) & ,-0.72)\end{array}$

SDI region

\begin{tabular}{|c|c|c|c|c|c|}
\hline High SDI & $\begin{array}{l}5.42(4.48 \\
, 5.72)\end{array}$ & $\begin{array}{l}0.65(0.54 \\
0.69)\end{array}$ & $\begin{array}{l}5.32(4.72 \\
, 5.8)\end{array}$ & $\begin{array}{l}0.42(0.38 \\
, 0.46)\end{array}$ & $\begin{array}{l}-1.58(-1.68 \\
,-1.49)\end{array}$ \\
\hline High-middle SDI & $\begin{array}{l}10.4(9.24 \\
, 12.26)\end{array}$ & $\begin{array}{l}0.93(0.83 \\
, 1.1)\end{array}$ & $\begin{array}{l}10.27(8.57 \\
, 11.27)\end{array}$ & $\begin{array}{l}0.7(0.58 \\
, 0.77)\end{array}$ & $\begin{array}{l}-0.9(-1.01 \\
,-0.78)\end{array}$ \\
\hline Middle SDI & $\begin{array}{l}13.45(10.21 \\
, 18.52)\end{array}$ & $\begin{array}{l}0.78(0.61 \\
, 1.05)\end{array}$ & $\begin{array}{l}17.08(13.05 \\
, 19.22)\end{array}$ & $\begin{array}{l}0.74(0.57 \\
, 0.83)\end{array}$ & $\begin{array}{l}0.14(0 \\
, 0.28)\end{array}$ \\
\hline Low-middle SDI & $\begin{array}{l}7.71(5.05 \\
, 12.78)\end{array}$ & $\begin{array}{l}0.6(0.42 \\
, 0.93)\end{array}$ & $\begin{array}{l}8.63(7.37 \\
, 10.4)\end{array}$ & $\begin{array}{l}0.5(0.43 \\
0.6)\end{array}$ & $\begin{array}{l}-0.61(-0.66 \\
,-0.57)\end{array}$ \\
\hline Low SDI & $\begin{array}{l}4.24(2.11 \\
, 9.66)\end{array}$ & $\begin{array}{l}0.6(0.35 \\
, 1.21)\end{array}$ & $6.32(4.7,8)$ & $\begin{array}{l}0.49(0.38 \\
, 0.6)\end{array}$ & $\begin{array}{l}-0.74(-0.79 \\
,-0.7)\end{array}$ \\
\hline
\end{tabular}

GBD region

\begin{tabular}{|c|c|c|c|c|c|}
\hline $\begin{array}{l}\text { High-income Asia } \\
\text { Pacific }\end{array}$ & $\begin{array}{l}1.17(0.95 \\
, 1.27)\end{array}$ & $\begin{array}{l}0.69(0.55 \\
0.75)\end{array}$ & $\begin{array}{l}1.01(0.78 \\
, 1.1)\end{array}$ & $\begin{array}{l}0.4(0.34 \\
0.44)\end{array}$ & $\begin{array}{l}-1.96 \\
(-2.09,-1.83)\end{array}$ \\
\hline $\begin{array}{l}\text { High-income North } \\
\text { America }\end{array}$ & $\begin{array}{l}1.88(1.59 \\
, 1.96)\end{array}$ & $\begin{array}{l}0.65(0.55 \\
0.67)\end{array}$ & $\begin{array}{l}2.13(1.86 \\
, 2.34)\end{array}$ & $\begin{array}{l}0.47(0.42, \\
0.52)\end{array}$ & $\begin{array}{l}-1.09 \\
(-1.16,-1.03)\end{array}$ \\
\hline Western Europe & $\begin{array}{l}2.57(2.07 \\
, 2.74)\end{array}$ & $\begin{array}{l}0.65(0.53, \\
0.7)\end{array}$ & $\begin{array}{l}2.36(2.05 \\
, 2.69)\end{array}$ & $\begin{array}{l}0.41(0.37 \\
0.46)\end{array}$ & $\begin{array}{l}-1.83 \\
(-1.93,-1.73)\end{array}$ \\
\hline Australasia & $\begin{array}{l}0.16(0.13 \\
0.17)\end{array}$ & $\begin{array}{l}0.78(0.64 \\
0.84)\end{array}$ & $\begin{array}{l}0.14(0.13 \\
, 0.17)\end{array}$ & $\begin{array}{l}0.42(0.38 \\
0.49)\end{array}$ & $\begin{array}{l}-2.61 \\
(-2.87,-2.36)\end{array}$ \\
\hline $\begin{array}{l}\text { Tropical Latin } \\
\text { America }\end{array}$ & $\begin{array}{l}1.38(1.23 \\
, 1.57)\end{array}$ & $\begin{array}{l}0.85(0.76 \\
0.97)\end{array}$ & $\begin{array}{l}1.5(1.27 \\
, 1.65)\end{array}$ & $\begin{array}{l}0.72(0.61 \\
0.8)\end{array}$ & $\begin{array}{l}-0.32 \\
(-0.48,-0.16)\end{array}$ \\
\hline $\begin{array}{l}\text { Andean Latin } \\
\text { America }\end{array}$ & $\begin{array}{l}0.49(0.38 \\
, 0.66)\end{array}$ & $\begin{array}{l}1.18(0.96, \\
1.56)\end{array}$ & $\begin{array}{l}0.82(0.55 \\
, 1.05)\end{array}$ & $\begin{array}{l}1.31(0.88, \\
1.68)\end{array}$ & $\begin{array}{l}0.55 \\
(0.39,0.7)\end{array}$ \\
\hline
\end{tabular}




\begin{tabular}{|c|c|c|c|c|c|}
\hline $\begin{array}{l}\text { Central Latin } \\
\text { America }\end{array}$ & $\begin{array}{l}2.27(2.1 \\
, 2.55)\end{array}$ & $\begin{array}{l}1.28(1.2 \\
1.47)\end{array}$ & $\begin{array}{l}3.66(2.92 \\
, 4.25)\end{array}$ & $\begin{array}{l}1.49(1.18 \\
1.73)\end{array}$ & $\begin{array}{l}0.78 \\
(0.65,0.91)\end{array}$ \\
\hline $\begin{array}{l}\text { Southern Latin } \\
\text { America }\end{array}$ & $\begin{array}{l}0.5(0.46 \\
, 0.55)\end{array}$ & $\begin{array}{l}1(0.92 \\
1.11)\end{array}$ & $\begin{array}{l}0.59(0.52 \\
, 0.64)\end{array}$ & $\begin{array}{l}0.87(0.77 \\
0.96)\end{array}$ & $\begin{array}{l}-0.36 \\
(-0.41,-0.32)\end{array}$ \\
\hline Caribbean & $\begin{array}{l}0.32(0.23 \\
, 0.46)\end{array}$ & $\begin{array}{l}0.87(0.65, \\
1.22)\end{array}$ & $\begin{array}{l}0.36(0.27 \\
, 0.48)\end{array}$ & $\begin{array}{l}0.8(0.58 \\
1.09)\end{array}$ & $\begin{array}{l}-0.16 \\
(-0.27,-0.05)\end{array}$ \\
\hline Eastern Europe & $\begin{array}{l}3.26(3.01 \\
, 3.72)\end{array}$ & $\begin{array}{l}1.48(1.35, \\
1.69)\end{array}$ & $\begin{array}{l}2.05(1.8 \\
, 2.35)\end{array}$ & $\begin{array}{l}0.86(0.76 \\
0.98)\end{array}$ & $\begin{array}{l}-2.15 \\
(-2.29,-2.01)\end{array}$ \\
\hline Central Europe & $\begin{array}{l}1.09(0.94 \\
, 1.19)\end{array}$ & $\begin{array}{l}0.89(0.77 \\
0.97)\end{array}$ & $\begin{array}{l}0.7(0.61 \\
0.81)\end{array}$ & $\begin{array}{l}0.51(0.44, \\
0.6)\end{array}$ & $\begin{array}{l}-1.87 \\
(-1.94,-1.8)\end{array}$ \\
\hline Central Asia & $\begin{array}{l}0.74(0.61 \\
, 0.88)\end{array}$ & $\begin{array}{l}1.02(0.86, \\
1.2)\end{array}$ & $\begin{array}{l}0.65(0.55 \\
, 0.78)\end{array}$ & $\begin{array}{l}0.73(0.61, \\
0.86)\end{array}$ & $\begin{array}{l}-1.23 \\
(-1.29,-1.18)\end{array}$ \\
\hline $\begin{array}{l}\text { North Africa \& } \\
\text { Middle East }\end{array}$ & $\begin{array}{l}3.19(1.96 \\
, 4.85)\end{array}$ & $\begin{array}{l}0.89(0.58 \\
1.29)\end{array}$ & $\begin{array}{l}3.61(2.53 \\
, 4.54)\end{array}$ & $\begin{array}{l}0.64(0.45, \\
0.8)\end{array}$ & $\begin{array}{l}-1.12 \\
(-1.16,-1.07)\end{array}$ \\
\hline South Asia & $\begin{array}{l}6.91(4.34 \\
, 12.22)\end{array}$ & $\begin{array}{l}0.54(0.36 \\
0.9)\end{array}$ & $\begin{array}{l}6.9(5.72 \\
, 8.49)\end{array}$ & $\begin{array}{l}0.39(0.32 \\
0.47)\end{array}$ & $\begin{array}{l}-1.45 \\
(-1.57,-1.34)\end{array}$ \\
\hline Southeast Asia & $\begin{array}{l}3.4(2.24 \\
, 5.5)\end{array}$ & $\begin{array}{l}0.7(0.49 \\
1.09)\end{array}$ & $\begin{array}{l}3.93(3.26 \\
, 4.94)\end{array}$ & $\begin{array}{l}0.63(0.52 \\
0.79)\end{array}$ & $\begin{array}{l}-0.34 \\
(-0.38,-0.3)\end{array}$ \\
\hline East Asia & $\begin{array}{l}8.62(6.08 \\
, 13.97)\end{array}$ & $\begin{array}{l}0.74(0.53, \\
1.19)\end{array}$ & $\begin{array}{l}12.14(8.08 \\
, 14.37)\end{array}$ & $\begin{array}{l}0.82(0.56 \\
0.96)\end{array}$ & $\begin{array}{l}0.94 \\
(0.56,1.31)\end{array}$ \\
\hline Oceania & $\begin{array}{l}0.03(0.02 \\
, 0.05)\end{array}$ & $\begin{array}{l}0.43(0.29 \\
0.65)\end{array}$ & $\begin{array}{l}0.05(0.03 \\
, 0.08)\end{array}$ & $\begin{array}{l}0.41 \text { (0.27, } \\
0.6)\end{array}$ & $\begin{array}{l}-0.36 \\
(-0.48,-0.24)\end{array}$ \\
\hline $\begin{array}{l}\text { Western Sub- } \\
\text { Saharan Africa }\end{array}$ & $\begin{array}{l}0.74(0.48 \\
, 1.1)\end{array}$ & $\begin{array}{l}0.31(0.22, \\
0.42)\end{array}$ & $1.5(1.1,2)$ & $\begin{array}{l}0.29(0.22 \\
0.38)\end{array}$ & $\begin{array}{l}-0.3 \\
(-0.37,-0.22)\end{array}$ \\
\hline $\begin{array}{l}\text { Eastern Sub- } \\
\text { Saharan Africa }\end{array}$ & $\begin{array}{l}2.22(0.93 \\
, 5.84)\end{array}$ & $\begin{array}{l}0.8(0.41 \\
1.86)\end{array}$ & $\begin{array}{l}3.01(1.81 \\
, 4.17)\end{array}$ & $\begin{array}{l}0.61(0.4, \\
0.8)\end{array}$ & $\begin{array}{l}-0.85 \\
(-0.97,-0.74)\end{array}$ \\
\hline $\begin{array}{l}\text { Central Sub- } \\
\text { Saharan Africa }\end{array}$ & $\begin{array}{l}0.22(0.09 \\
, 0.49)\end{array}$ & $\begin{array}{l}0.3(0.15 \\
0.58)\end{array}$ & $\begin{array}{l}0.37(0.24 \\
, 0.58)\end{array}$ & $\begin{array}{l}0.25(0.17 \\
0.37)\end{array}$ & $\begin{array}{l}-0.48 \\
(-0.6,-0.35)\end{array}$ \\
\hline $\begin{array}{l}\text { Southern Sub- } \\
\text { Saharan Africa }\end{array}$ & $\begin{array}{l}0.11(0.09 \\
, 0.15)\end{array}$ & $\begin{array}{l}0.2(0.16 \\
0.26)\end{array}$ & $\begin{array}{l}0.15(0.12 \\
, 0.19)\end{array}$ & $\begin{array}{l}0.19(0.15 \\
0.24)\end{array}$ & $\begin{array}{l}-0.04 \\
(-0.12,0.03)\end{array}$ \\
\hline
\end{tabular}

When comparing regions, the most deaths occurred in the middle SDI region (17 080 deaths in 2019), with the highest ASDR (ASDR: 0.74, 95\% Cl 0.57 0.83) and fastest-growing ASDR (EACP: $0.14,95 \% \mathrm{Cl}$ $0 \sim 0.28)$. The other four regions showed a decreased tendency. Subgroup analysis of geographical zone indicated that East Asia (8 620 cases) and South Asia (6 910 cases) had the most deaths, with a positive ASDR of 0.94 and a negative ASDR of 1.45, respectively. Also, East Asia had the fastest rise in ASDR 
$(E A P C=0.94,95 \% \mathrm{Cl} 0.56 \sim 1.31)$, while Australia and Eastern Europe had a remarkable decline trend (EAPC of Australia: -2.61, 95\% Cl-2·87 -2.36; of Eastern Europe: -2.15, 95\% Cl-2.29 -2.01; Table 2).

When we compared countries or territories, China and India were the top 2 countries with the most deaths (China: 11873 in 2019; India: 4505 in 2019) (Additional file 2: Table S4). Ecuador had the highest ASDR $(1.69 / 100$ 000) in 2019, while this highest value was 2.22 in Ukraine in 1990 (Fig. 1B, Additional file 2: Table S5, S6). Guatemala had the most rapid increase in ASDR (EAPC: 7.51, 95\% Cl 6.67 8.35), and it also had a remarkable increase in El Salvador, Lesotho, and Taiwan (Province of China; Fig. 1D, Additional file 2: Table S7).

\section{DALYs tendency and variation in ALL}

Globally, compared with 1990, the number of DALYs decreased by about 69000 in 2019, with $27.3 \times 10^{5}$ in 1990 and $26.61 \times 10^{5}$ in 2019. Age-standardized DALYs rate decreased by 0.81 per year (Additional file 2: Table S1). Males were likely the main contributor to ALL DALYs rate. Females not only had a lower agestandardized DALYs rate, but also had a more rapid decrease in this rate (DALYs in 2019, male: $16.07 \times 10^{5}$, female: $10.54 \times 10^{5}$; EAPC of age-standardized DALYs rate, male: -0.65 , female: -1.06 ; Table S1, Additional file 1: Fig. S1C).

Subgroup analysis of SDI regions demonstrated that both the highest-burden and lowest decrease of DALYs occurred in the middle SDI region (DALYs: $9.25 \times 10^{5}$ in 2019, age-standardized DALYs rate: 41.47/100 000 in 2019; EAPC: $-0.24,95 \% \mathrm{Cl}$ :-0.37 -0.11). The ASR of DALYs in the high SDI, high-middle SDI, low-middle SDI, and low SDI regions gradually decreased by $1.75,1.13,0.98$, and 0.89 , respectively.

Comparing GBD region levels, similar to deaths, East Asia had the highest DALYs both in 1990 and 2019 (5.61× $\times 10^{5}$ in 1990 and $5.88 \times 10^{5}$ in 2019), followed by South Asia. Almost all regions had a negative EAPC except Andean Latin America, Central Latin America, and East Asia. Thereinto, Australasia had the most notable decrease in ASR of DALYs (EAPC: -2.78, 95\% Cl: -3.02 -2.54; Additional file 2: Table S1). Promisingly, there were five countries' age-standardized DALYs rate more than 100 (Ukraine: 145.937, Ethiopia, Belarus, Honduras, and Moldova) in 1990, but the highest age-standardized DALYs rate in 2019 was under 100 (Mexico, 98.25, followed by Ecuador, 97.7; Additional file 1: Fig. S4A, Additional file 2: Table S8 and S9). Guatemala's age-standardized DALYs rate grew fastest over the past 30 years (EAPC = 7.33, 95\% Cl 6.5 8.18; Additional file 1: Fig. S5B, Additional file 2: Table S10).

\section{Influential factors for EAPCs of ALL burden}

We found a significant positive correlation between the EAPC of ASIR and baseline ASIR in 1990 ( $\rho=$ $0.167, P=0.017)$ and SDI in $2019(\rho=0.357, P=1.7 e-07)$ at the national level (Fig. 2A-2B). However, the reverse relationship between EAPC of ASDR and baseline ASDR in $1990(\rho=-0.401, P=2.6 \mathrm{e}-09)$ and SDI in $2019(\rho=-0.596, P<2.2 \mathrm{e}-16)$ were observed (Fig. 2C-2D). Albeit the ASIR increased significantly in most 
countries and territories with SDI more than 0.7 in 2019, the corresponding ASDR dropped obviously, which indicated the treatment protocol in high SDI region would be worth learning and promoting in other areas. For EAPC of DALYs, a similar characteristic to ASDR is shown in Additional file (Figure S3).

We then investigated the correlation between SDI and ASIR, ASDR, and age-standardized DALYs rate in 21 GBD regions. Results showed that annual ASIR had remained relatively stable with SDI progressing in most low-burden GBD regions; however, the ASIR presented a steep increase in most high-burden GBD regions, especially East Asia, Western Europe, and High-income Asia Pacific (Fig. 3A). Inversely, the ASDR and age-standardized DALYs rate markedly dropped with SDI development in most GBD regions, especially in high SDI region. Notably, the ASDR and age-standardized DALYs rate rose beforehand and decreased afterward in Central Latin America and Andean Latin America (Fig. 3B, Additional file 1: Fig. S6).

\section{Age characters of ALL}

We divided the population into 20 groups according to ages in the global and SDI regional areas: patients aged 0 90 years old were divided into 19 groups, with a component gap of 5-years, and the final group was above 95 years old. Globally, children aged 0 9 years old and the elderly were the most vulnerable populations according to incidence and death rates (Additional file 1: Fig. S7A-S7C), but which presented an obvious disparity referring to SDI regions (Fig. 4A-4B, Additional file 1: Fig. S8). In low-middle and low SDI regions, the ALL burden focused on the population under 25 years old. Conversely, the proportion of patients aged 25 years and older increased gradually from 1990 to 2019 among both sexes in the remained three SDI regions (Fig. 4A, Additional file 1: Fig. S8). In addition, the incidence rate of ALL in young people usually decreased, but increased among the elderly, whose ASIR had almost twice as many as children in 2019 (Fig. 5A, Additional file 1: Fig. S9A). Death rate and DALYs rate in children remained high, but the older patients' death rate became much higher, even exceeding children, showing a positive correlation with age (Fig. 5B, Additional file 1: Fig. S9B-S9C, S10). The rate of increase in adults was also the fastest; EAPCs of 25-69 years old patients were around 2.5 from Additional file (Figure S2A). Particularly, middle SDI region patients (both sexes) over 60 years old had a higher EAPC in agestandardized death/DALYs rate, while EAPC in children was under 0 , presenting hopeful treatment strategies (Additional file 1: Fig. S3B-S3C, S2).

\section{Risk factors contributing to ALL-related deaths or DALYs}

Smoking was the predominant contributor to ALL-related DALYs for males across the world, while high body mass index was the main cause of ALL-related DALYs for females in 2019 (Fig. 6A-6C). In high SDI region, the role of smoking in females ALL-related DALYs was greater than the role of high body mass index (Fig. 6B-6C). Occupational exposure to formaldehyde gradually became an important factor from 1990 to 2019, especially in middle, low-middle, and low SDI regions (Fig. 6). Subgroup analysis of the 
GBD geographic zone suggested that the ratio of high body mass index accounted for an increasing proportion of all risk factors from 1990 to 2019 (Additional file 1: Fig. S11).

\section{Discussion}

In our study, we comprehensively analyzed the global, regional, and national burden in incidence, deaths, and DALYs of ALL along with the temporal trend over the past 30 years in 204 countries and territories. Globally, ALL incidence and deaths have increased, with an increased ASIR and a decreased ASDR, respectively. But both DALYs and ASR of DALYs were declined. Before our study, two articles have analyzed the global burden of ALL based on the database of GBD 2017 (Ou et al. 2020; Ming Yi 2020). whose results differed from our study in some aspects due to different methods or differential data sources of GBD 2017. The latest methods and data sources based on GBD 2019 made some optimizations and updates compared to GBD 2017. First, GBD 2019 added the updated cancer mortality, which made the estimation on ALL reconstructed by mathematical models more robust. Second, to acquire more training data and less smoothing across time and geography, the mortality-to-incidence ratio estimation was updated with lower case inclusion criteria and different model hyperparameters. And third, the covariates used in CODEm models were adjusted for GBD 2019. These modulations make our study more comprehensive and precise. For example, the GBD 2017 illustrated almost no change for ASIR of around $0.87 / 100000$, but the ASIR was increased by 1.61 per year from 1.23/100 000 in 1990 to $1.96 / 100000$ in 2019 in our results.

Our findings demonstrated the incidence cases of ALL increased by $1.29 \%$ in the past 30 years. That might be due to the change in its diagnostic criteria (The diagnostic criteria for bone marrow blasts decreased from $\geq 30 \%$ to $\geq 20 \%$ ), or because more and more doctors started to recognize and diagnose ALL at the early stage. Our data also suggested that we have made great progress in treatment and management, but much remains to be done in prevention. However, the current burden and change trend of ALL varies greatly with more than 100 times across the countries and territories, which makes the ALL's burden control more complex. Notably, in most high burden areas of ALL, the growth trend was more obvious, especially in East Asia and Western Europe. Therefore, understanding the exact pattern of the causes of ALL is key to prevention and treatment.

According to our studies, male patients were more likely to be suffering from ALL than females, and the difference between sexes further widened from 1990 to 2019. Geographically, middle SDI region had the highest incidence and the most rapid ASIR. We found that Western Europe and high-income Asia Pacific regions had the highest ASIR, while Latin America had the highest ASDR and age-standardized DALYS rate, for reasons that have not yet been studied previously. Nationally, when studying the correlation between SDI and ASIR, ASDR and age-standardized DALYs rate, we found a significant positive correlation between the EAPC of ASIR and baseline ASIR in 1990 and SDI in 2019. However, the converse relationship between EAPC of ASDR and baseline ASDR in 1990 and SDI in 2019 were observed. This reflects the disparate problem as trends in morbidity and mortality. It may be that in these middle or lowmiddle areas that industrialization has led to a rise in environmental carcinogens, like benzene. But the 
prognosis of ALL has been improved greatly in developed countries because of improved medical care and affordable treatment.

In most regions, patients aged 0 9 years still have a higher incidence. Based on previous work, inherited genetic variants probably contributed directly to a genetic vulnerability of hemopoietic cells (Florent Malard 2020; Trevino et al. 2009). IKZF1, ARID5B, CEBPE, CDKN2A, ETV6, and PAX5 were believed to significantly and consistently be associated with childhood ALL (Gocho and Yang 2019; Sherborne et al. 2010; Xie et al. 2014; Carl H. June 2018; June and Sadelain 2018; Michel Sadelain 2015; Kantarjian et al. 2017). Environmental factors like pesticide exposure, ionizing radiation and childhood infections are also important contributors to ALL's occurrence. Therefore, attention should be paid to the environmental problems caused by industrial development especially in low-middle and low SDI areas.

However, with the global aging population, in the 25-69 years old patients, a gradual increase was seen in incidence cases and ASIR from 1990 to 2019. Aging has previously been associated with a functional decline of hematopoietic stem cells (HSC) as well as an increased risk of myeloid malignancies (Liu et al. 2019). In 2019, patients over 50 years of age accounted for almost half of all patients in the high SDI region, which may due to a long lifespan. A long lifespan could increase the incidence of many diseases, not only ALL. And this proportion represented a small percentage of patients in low-middle and low SDI regions. This may be due to the reduced economy and fewer medical options and an incomplete registration system in low SDI region. However, based on the data we have, health management of older adults should be a priority in high, high-middle, and middle SDI areas. In this situation, older patients' worse prognosis is also an obstacle of ALL's treatment. In recent years, the development of novel drugs, such as blinatumomab, rituximab, tyrosine kinase inhibitors, CAR-T cells, and others, have improved the prognosis of both adolescents and young adults, adults and older adults (Florent Malard 2020; Chunxia et al. 2019; Jenkins et al. 2017; Fuentes et al. 2010; Nunez-Enriquez et al. 2019; Orgel, Sea, and Mittelman 2019). We believe these novel therapies will provide a bright landscape for ALL treatment.

Based on the GBD database, we analyzed four possible factors attribute to ALL-related deaths and DALYs. Smoking was the first and second reason in males and females in 2019, respectively. According to the World Health Organization (WHO), there were 1.3 billion smokers in the world, and men were five times more likely than women to smoke. A meta-analysis in 2019 reported that paternal smoking was significantly associated with the elevated risk of childhood ALL during pregnancy, but maternal smoking was not (Goldstein 2010). No studies have linked smoking with morbidity and mortality in older patients. Tobacco smoke contains more than 4000 chemicals including a myriad of known carcinogens. It has been reported that smoking is associated with an accumulation of cadmium and lead in seminal plasma, reduced sperm count and motility, and fewer morphologically normal sperm (Heck et al. 2019; Jiang, Wu Sy Fau - Ke, and Ke 2016).

For females, high body mass index was the first contributor to ALL-related deaths and DALYs. A large cohort study in Mexican children suggested that overweight and obese patients embody a subgroup with a high risk of dying during leukemia treatment (Jabo et al. 2017). The mechanisms of obesity leading to 
ALL may include (1) hyperinsulinemia associated with obesity-induced insulin resistance, (2) interstitial levels of adipocyte-released fuels that are likely extremely high in the adipose tissue microenvironment, and/or (3) obesity associated with chronic inflammation, a well-known contributor to solid tumor oncogenesis (Orgel, Sea, and Mittelman 2019). Notably, with the improvement of life quality, high body mass index gradually accounted for a bigger proportion of all factors. Therefore, we should focus more on the dietary structure of ALL patients, especially in high SDI region.

Benzene, a chemical reagent, has been documented to lead to a broad range of genotoxic effects in lymphocytes of benzene-exposed workers, and has chromosomal effects in carcinogenesis (Goldstein 2010). A population-based study identified that maternal occupational exposure to benzene during pregnancy was related to increased risk of ALL in offspring (adjusted OR $=2.28,95 \% \mathrm{Cl} 1.17$ to 4.41 ) (Heck et al. 2019). As similar to benzene, it has been reported that the median formaldehyde and benzene concentrations in ALL $(32.120 \mathrm{pg} / \mathrm{ml})$ were significantly higher than those in controls $(18.705 \mathrm{pg} / \mathrm{ml})$ (Jiang, Wu Sy Fau - Ke, and Ke 2016). Notably, occupational exposure to formaldehyde gradually became an important factor from 1990 to 2019, especially in the low-middle and low SDI regions, where industrialization is developing rapidly. This finding may remind policy-makers to prioritize environmental protection as well as development.

Besides the above-mentioned risk factors, neighborhood socioeconomic status (nSES), marital status and race would influence ALL treatment. It has been reported that lower nSES quintiles predicted lower chemotherapy and hematopoietic cell transplantation utilization for ALL. Compared to married patients, never married patients had a lower RR of receiving chemotherapy or hematopoietic cell transplantation. Lower HCT utilization occurred in Hispanic and non-Hispanic black patients when compared to nonHispanic whites, which explained racial differences (Jabo et al. 2017).

To our knowledge, we reported the latest burden of ALL across the world, which provides an evidencebased assessment for the formulation of ALL management strategies at the global, regional, and even national levels. However, some limitations need to be mentioned in our study. Firstly, the reconstructed data may be not accurate in some countries with sparse prior information, but the robust estimation performed by the GBD study has somewhat alleviated this concern. Moreover, the specific pathogenesis and treatment for ALL need further basic research and clinical doctor's efforts. Finally, many attributions of risk factors are associated with ALL-related mortality, here we only analyzed the available four risk factors due to the restriction of the GBD study, and other risk factors require further analysis in the future study. In any case, the global burden of ALL is still increasing, and we should take active measures to minimize the harm of ALL to human beings.

\section{Conclusions}

Globally, the incidence burden was heavier than mortality. Although people aged $0 \sim 9$ years were more likely to suffer from ALL, the increasing number of middle-aged and older patients cannot be ignored. Older adults accounted for a bigger proportion of all patients and had the worse prognosis; and given the 
population is aging, the global burden of ALL is becoming heavier. Both the highest incidence and fastest ASIR occurred in the middle SDI region; genetic screening and avoidance of infection need to be addressed in these areas. Smoking, high body mass index, occupational exposure to benzene, and occupational exposure to formaldehyde were the main risk factors contributing to ALL-related mortality. We need to control occupational exposure to carcinogens, especially in developing countries. But for developed countries, body mass index needs to be taken seriously. Through our analysis, policy-makers can develop policies to reduce the burden of ALL on a regional basis.

\section{Abbreviations}

ALL, acute lymphoblastic leukemia; EAPC, estimated annual percentage change; SDI, sociodemographic index; DALY, disability-adjusted life year; GBD, Global Burden of Disease; GHDx, Global Health Data Exchange; ASR: age-standardized rate; ASIR: age-standardized incidence rate; ASDR: age-standardized death rate.

\section{Declarations}

\section{Acknowledgements}

We would like to thank the countless individuals who have contributed to the Global Burden of Disease Study 2019 in various capacities. This work was supported by National Natural Science Foundation of China (No. 81300383, No. 81900121), Major Research and Development Plan of Shandong Province (No. 2019GSF108240), Young Taishan Scholar Foundation of Shandong Province (No. tsqn202103167, No. tsqn201909175), and Clinical Research Center of Shandong University (No. 2020SDUCRCC015, No. 2020SDUCRCC009). The authors also acknowledge that Alexandra Marshall of Marshall Medical Communications, provided editorial assistance.

\section{Competing interests}

The authors declare no potential competing interest.

\section{Availability of data and materials}

The datasets generated during and/or analyzed during the current study are available from the Global Health Data Exchange query tool, http://ghdx.healthdata.org/gbd-results-tool.

\section{Code availability}

R 4.0.3 


\section{Authors' contributions}

Conceptualization: Xiaorong Yang, Shuqian Xu; Methodology: Xiaorong Yang, Yuefen Hu; Formal analysis and investigation: Yufen Hu, Xiuping Zhang, Aijun Zhang; Data Curation: Qizhao Li, Yafei Yu; Writing original draft preparation: Yuefen Hu; Writing - review and editing: Jun Peng, Ming Hou, Shuqian Xu; Funding acquisition: Shuqian Xu, Yu Hou; Supervision: Yang Liu, Yawen Wang; Visualization: all authors.

\section{Ethics approval}

The GBD 2019 study is a publicly available database and all data were anonymous. Our research protocol was approved by the Institutional Review Boards of Qilu Hospital of Shandong University with approval number KYLL-202011(KS)-239.

\section{ORCID}

Shuqian Xu https://orcid.org/0000-0001-7071-8550

\section{References}

1. Carl H. June Roddy S. O'Connor, Omkar U. Kawalekar,Saba Ghassemi,Michael C. Milone (2018) CAR T cell immunotherapy for human cancer.https://doi.org/10.1126/science.aar6711

2. Chunxia D., Meifang W., Jianhua Z., Ruijuan Z., Xiue L., Zhuanzhen Z., and Linhua Y. (2019) Tobacco smoke exposure and the risk of childhood acute lymphoblastic leukemia and acute myeloid leukemia: A meta-analysis. Medicine (Baltimore), 98: e16454.https://doi.org/10.1097/MD.0000000000016454

3. Collaborators GBD 2019 Diseases and Injuries (2020a) Global burden of 369 diseases and injuries in 204 countries and territories, 1990-2019: a systematic analysis for the Global Burden of Disease Study 2019. Lancet. 1204-22.https://doi.org/10.1016/S0140-6736(20)30925-9

4. Collaborators GBD 2019 Risk Factors (2020b) Global burden of 87 risk factors in 204 countries and territories, 1990-2019: a systematic analysis for the Global Burden of Disease Study 2019. Lancet. 1223-49.https://doi.org/10.1016/S0140-6736(20)30752-2

5. Dong Ying, Shi Oumin, Zeng Quanxiang, Lu Xiaoqin, Wang Wei, Li Yong, and Wang Qi (2020) Leukemia incidence trends at the global, regional, and national level between 1990 and 2017. Experimental Hematology \& Oncology, 9.https://doi.org/10.1186/s40164-020-00170-6

6. Florent Malard Mohamad Mohty (2020) Acute lymphoblastic leukaemia. Lancet. 114662.https://doi.org/10.1016/S0140-6736(19)33018-1

7. Fuentes A., Munoz A., Barnhart K., Arguello B., Diaz M., and Pommer R. (2010) Recent cigarette smoking and assisted reproductive technologies outcome. Fertil Steril, 93: 8995.https://doi.org/10.1016/j.fertnstert.2008.09.073 
8. Gocho Y., and Yang J. J. (2019) Genetic defects in hematopoietic transcription factors and predisposition to acute lymphoblastic leukemia. Blood, 134: 793-

97.https://doi.org/10.1182/blood.2018852400

9. Goldstein B. D. (2010) Benzene as a cause of lymphoproliferative disorders. Chem Biol Interact, 184: 147-50.https://doi.org/10.1016/j.cbi.2009.12.021

10. Gregory S. (2019) Adult Acute Lymphoblastic Leukemia: Treatment and Management Updates. Semin Oncol Nurs, 35: 150951.https://doi.org/10.1016/j.soncn.2019.150951

11. Heck J. E., He D., Contreras Z. A., Ritz B., Olsen J., and Hansen J. (2019) Parental occupational exposure to benzene and the risk of childhood and adolescent acute lymphoblastic leukaemia: a population-based study. Occup Environ Med, 76: 527-29.https://doi.org/10.1136/oemed-2019105738

12. Inaba Hiroto, Greaves Mel, and Mullighan Charles G. (2013) Acute lymphoblastic leukaemia. The Lancet, 381: 1943-55.https://doi.org/10.1016/s0140-6736(12)62187-4

13. Jabo B., Morgan J. W., Martinez M. E., Ghamsary M., and Wieduwilt M. J. (2017) Sociodemographic disparities in chemotherapy and hematopoietic cell transplantation utilization among adult acute lymphoblastic and acute myeloid leukemia patients. PLoS One, 12: e0174760.https://doi.org/10.1371/journal.pone.0174760

14. Jenkins T. G., James E. R., Alonso D. F., Hoidal J. R., Murphy P. J., Hotaling J. M., Cairns B. R., Carrell D. T., and Aston K. I. (2017) Cigarette smoking significantly alters sperm DNA methylation patterns. Andrology, 5: 1089-99.https://doi.org/10.1111/andr.12416

15. Jiang W. C., Wu Sy Fau - Ke Y. B., and Ke Y. B. (2016) [Association of exposure to environmental chemicals with risk of childhood acute lymphocytic leukemia]

16. Juliusson G., and Hough R. (2016) Leukemia. Prog Tumor Res, 43: 87100.https://doi.org/10.1159/000447076

17. June C. H., and Sadelain M. (2018) Chimeric Antigen Receptor Therapy. N Engl J Med, 379: 6473.https://doi.org/10.1056/NEJMra1706169

18. Kantarjian H., Stein A., Gokbuget N. et al. (2017) Blinatumomab versus Chemotherapy for Advanced Acute Lymphoblastic Leukemia. N Engl J Med, 376: 836-

47.https://doi.org/10.1056/NEJMoa1609783

19. Linker C. A., Levitt L. J., O'Donnell M., Ries C. A., Link M. P., Forman S. J., and Farbstein M. J. (1987) Improved results of treatment of adult acute lymphoblastic leukemia. Blood, 69: 124248.https://doi.org/10.1182/blood.V69.4.1242.1242

20. Liu D., Zhao J., Song Y., Luo X., and Yang T. (2019) Clinical trial update on bispecific antibodies, antibody-drug conjugates, and antibody-containing regimens for acute lymphoblastic leukemia. $J$ Hematol Oncol, 12: 15.https://doi.org/10.1186/s13045-019-0703-z

21. Michel Sadelain Renier Brentjens, Isabelle Rivière, Jae Park (2015) CD19 CAR Therapy for Acute Lymphoblastic Leukemia.https://doi.org/10.14694/EdBook_AM.2015.35.e360 
22. Ming Yi Linghui Zhou, Anping Li, Suxia Luo, Kongming Wu (2020) Global burden and trend of acute lymphoblastic leukemia from 1990 to 2017.https://doi.org/10.18632/aging.103982

23. 'National Cancer Institute. Cancer Stat Facts: Leukemia - Acute Lymphocytic Leukemia (ALL)'. https://seer.cancer.gov/statfacts/html/alyl.html.

24. Network National Comprehensive Cancer NCCN Clinical Practice Guidelines in Oncology: Acute Lymphoblastic Leukemia

25. Nunez-Enriquez J. C., Gil-Hernandez A. E., Jimenez-Hernandez E. et al. (2019) Overweight and obesity as predictors of early mortality in Mexican children with acute lymphoblastic leukemia: a multicenter cohort study. BMC Cancer, 19: 708.https://doi.org/10.1186/s12885-019-5878-8

26. Orgel E., Sea J. L., and Mittelman S. D. (2019) Mechanisms by Which Obesity Impacts Survival from Acute Lymphoblastic Leukemia. J Natl Cancer Inst Monogr, 2019: 152-

56.https://doi.org/10.1093/jncimonographs/lgz020

27. Ou Z., Yu D., Liang Y., He W., Li Y., Zhang M., You F., He H., and Chen Q. (2020) Analysis of the Global Burden of Disease study highlights the trends in death and disability-adjusted life years of leukemia from 1990 to 2017. Cancer Commun (Lond), 40: 598-610.https://doi.org/10.1002/cac2.12094

28. Pourshams Akram, Sepanlou Sadaf G., Ikuta Kevin S. et al. (2019) The global, regional, and national burden of pancreatic cancer and its attributable risk factors in 195 countries and territories, 19902017: a systematic analysis for the Global Burden of Disease Study 2017. The Lancet Gastroenterology \& Hepatology, 4: 934-47.https://doi.org/10.1016/s2468-1253(19)30347-4

29. Pui C. H., Campana D., Pei D. et al. (2009) Treating childhood acute lymphoblastic leukemia without cranial irradiation. N Engl J Med, 360: 2730-41.https://doi.org/10.1056/NEJMoa0900386

30. Ronson A., Tvito A., and Rowe J. M. (2016) Treatment of Relapsed/Refractory Acute Lymphoblastic Leukemia in Adults. Curr Oncol Rep, 18: 39.https://doi.org/10.1007/s11912-016-0519-8

31. Sherborne A. L., Hosking F. J., Prasad R. B. et al. (2010) Variation in CDKN2A at 9p21.3 influences childhood acute lymphoblastic leukemia risk. Nat Genet, 42: 492-4.https://doi.org/10.1038/ng.585

32. Terwilliger T., and Abdul-Hay M. (2017) Acute lymphoblastic leukemia: a comprehensive review and 2017 update. Blood Cancer J, 7: e577.https://doi.org/10.1038/bcj.2017.53

33. Trevino L. R., Yang W., French D. et al. (2009) Germline genomic variants associated with childhood acute lymphoblastic leukemia. Nat Genet, 41: 1001-5.https://doi.org/10.1038/ng.432

34. Veerman Anjo J., Kamps Willem A., van den Berg Henk et al. (2009) Dexamethasone-based therapy for childhood acute lymphoblastic leukaemia: results of the prospective Dutch Childhood Oncology Group (DCOG) protocol ALL-9 (1997-2004). The Lancet Oncology, 10: 95766.https://doi.org/10.1016/s1470-2045(09)70228-1

35. Vrooman L. M., and Silverman L. B. (2016) Treatment of Childhood Acute Lymphoblastic Leukemia: Prognostic Factors and Clinical Advances. Curr Hematol Malig Rep, 11: 38594.https://doi.org/10.1007/s11899-016-0337-y

36. Xie M., Lu C., Wang J. et al. (2014) Age-related mutations associated with clonal hematopoietic expansion and malignancies. Nat Med, 20: 1472-8.https://doi.org/10.1038/nm.3733 
37. Yang X., Man J., Chen H., Zhang T., Yin X., He Q., and Lu M. (2021) Temporal trends of the lung cancer mortality attributable to smoking from 1990 to 2017: A global, regional and national analysis. Lung Cancer, 152: 49-57.https://doi.org/10.1016/j.lungcan.2020.12.007

\section{Figures}

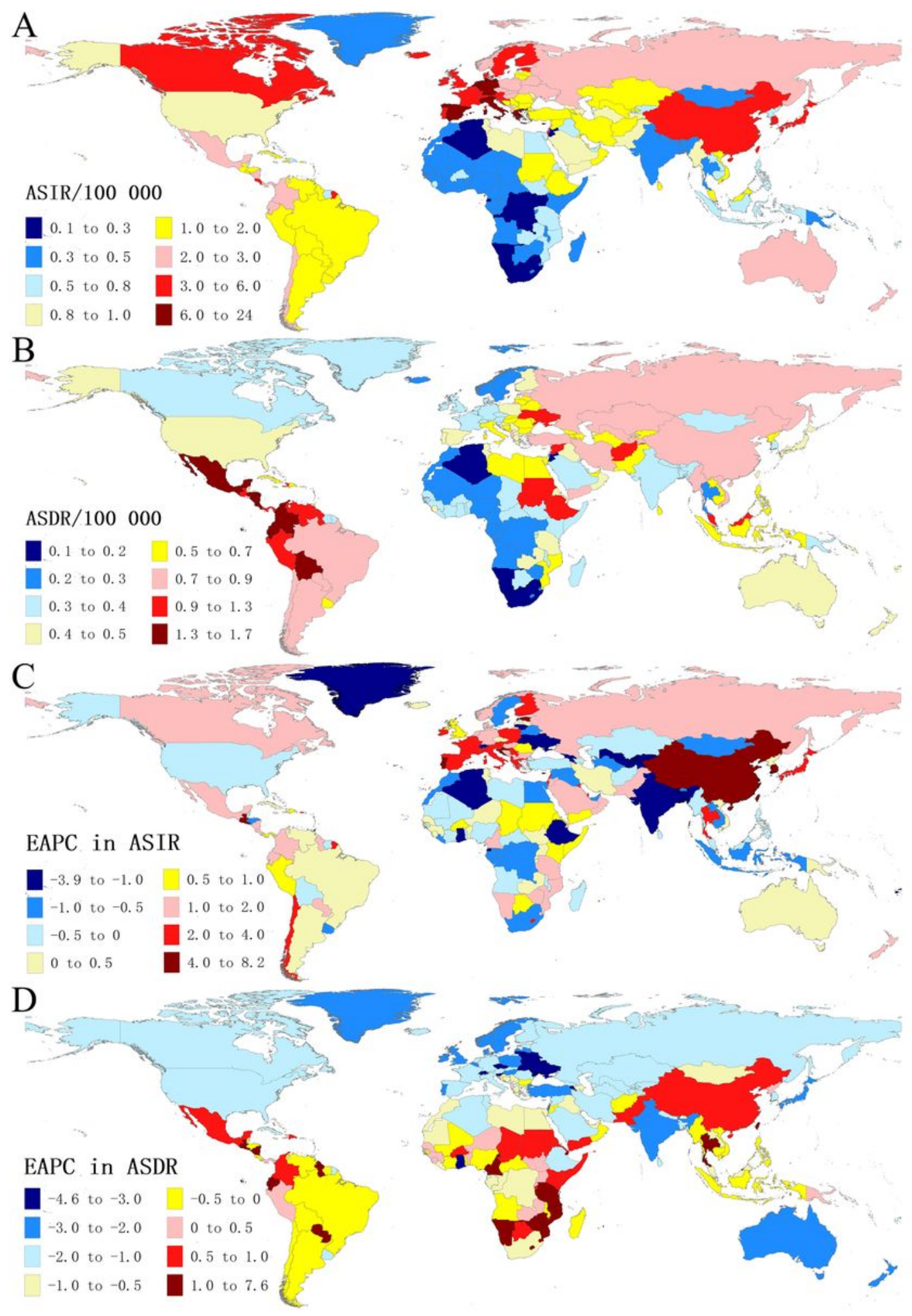

Figure 1 
Age-standardized rate of incidence and death and change trend of ALL in 204 countries or territories in 2019. A: ASIR, B: ASDR, C: EAPC in ASIR, D: EAPC in ASDR. Note: The designations employed and the presentation of the material on this map do not imply the expression of any opinion whatsoever on the part of Research Square concerning the legal status of any country, territory, city or area or of its authorities, or concerning the delimitation of its frontiers or boundaries. This map has been provided by the authors.


Figure 2 
Correlation analyses of EAPCs-ASR (1990) and EAPCs-SDI (2019) in 204 countries or territories. A) Correlation between EAPC of ASIR and baseline ASIR of 1990. B) Correlation between EAPC of ASIR and SDI of 2019. C) Correlation between EAPC of ASDR and baseline ASDR of 2019. D) Correlation between EAPC of ASDR and SDI of 2019. The size of circle represents the quantity of ALL patients in one country or territory.
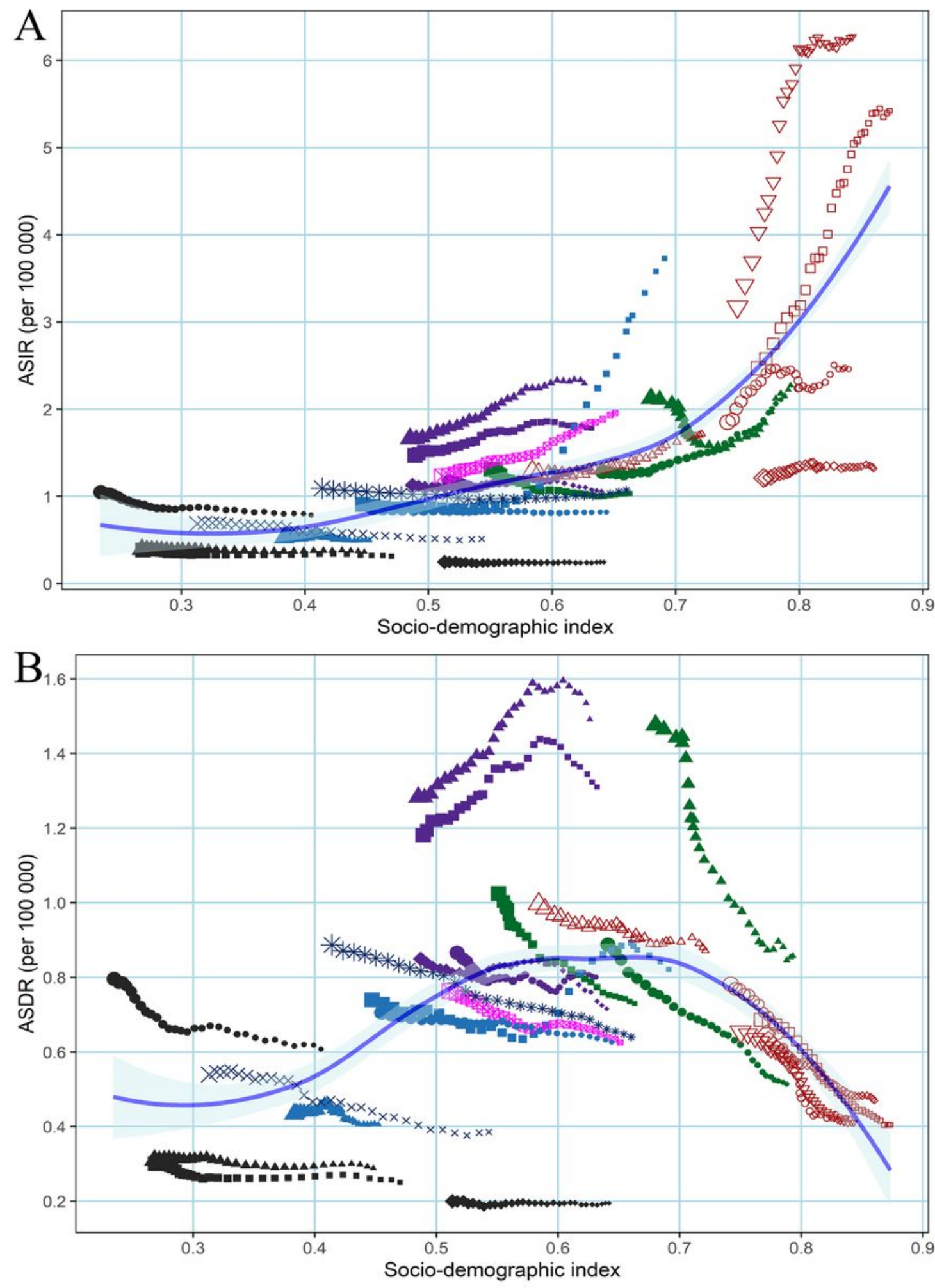

Year

- 1990

- 1995

- 2000

- 2005

- 2010

- 2015

- 2019

\section{GBD regions}

凶 Global

- Central Sub-Saharan Africa

- Eastern Sub-Saharan Africa

- Western Sub-Saharan Africa

- Southern Sub-Saharan Africa

* North Africa and Middle East

$\times$ South Asia

- East Asia

- Southeast Asia

- Oceania

- Andean Latin America

- Caribbean

- Central Latin America

- Tropical Latin America

- Central Asia

- Central Europe

- Eastern Europe

- Australasia

High-income Asia Pacific

- High-income North America

$\Delta$ Southern Latin America

$\nabla$ Western Europe

\section{Figure 3}


Change trends and correlation analyses of ASIR/ASDR and SDI from 1990 to 2019 in 21 regions. A) Change trends and correlation of ASIR and SDI. B) Change trends and correlation of ASDR and SDI.


Age groups

- $0-4$

5-9 $10-14$
$15-19$
$20-24$ $20-24$
$25-29$ $25-29$
$30-34$ 35-39 40-44 50-54 $50-54$
$55-59$ $\begin{array}{r}55-59 \\ -60-64 \\ \hline\end{array}$ $60-64$
$65-69$ 70-74 $75-79$
$80-84$
$85-89$ $85-89$
$90-94$

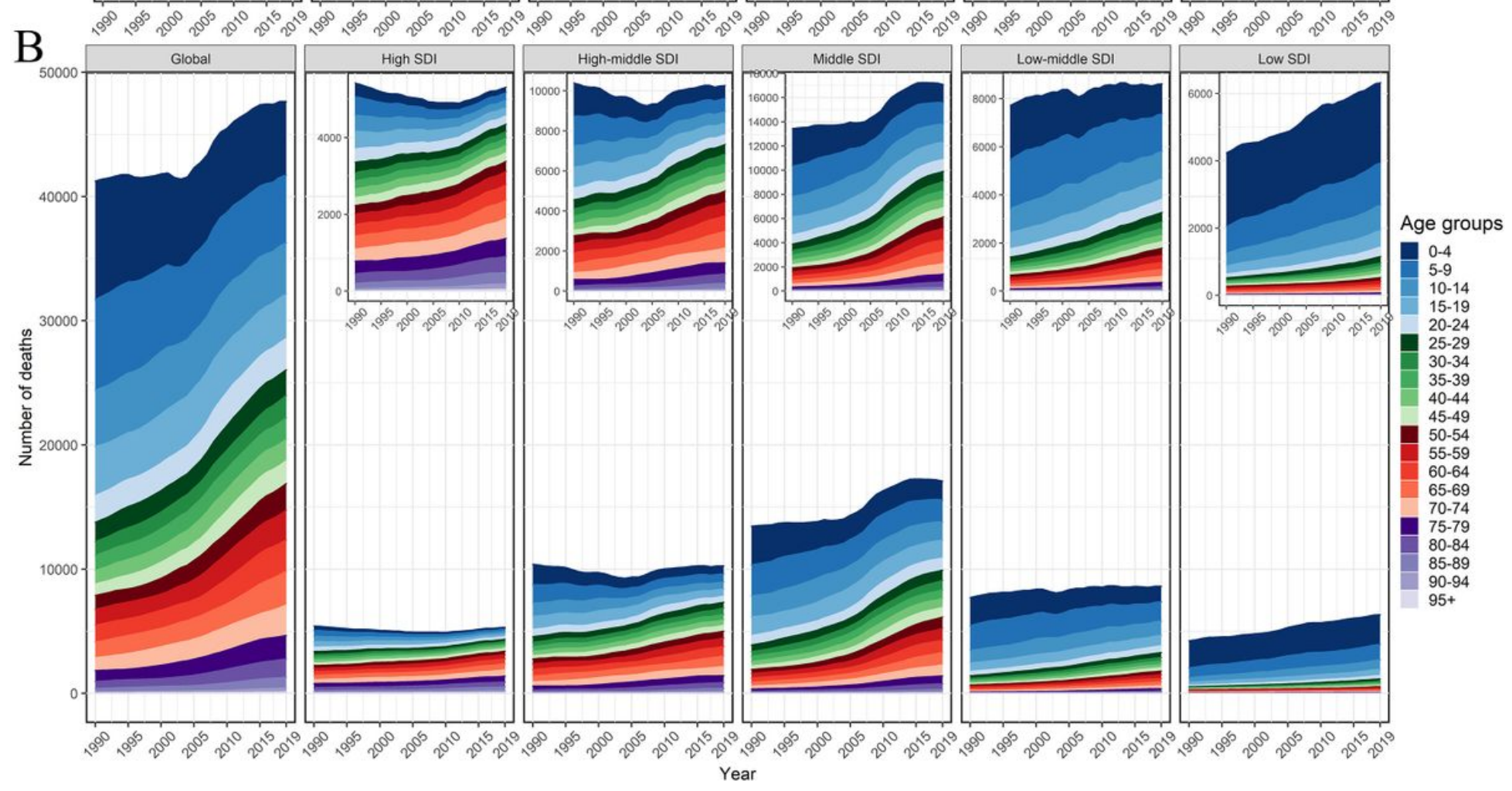

Figure 4

Proportion of different sex and age groups in the total number of cases by globe and SDI regions from 1990 to 2019. A) Incidence cases. B) Death cases. 


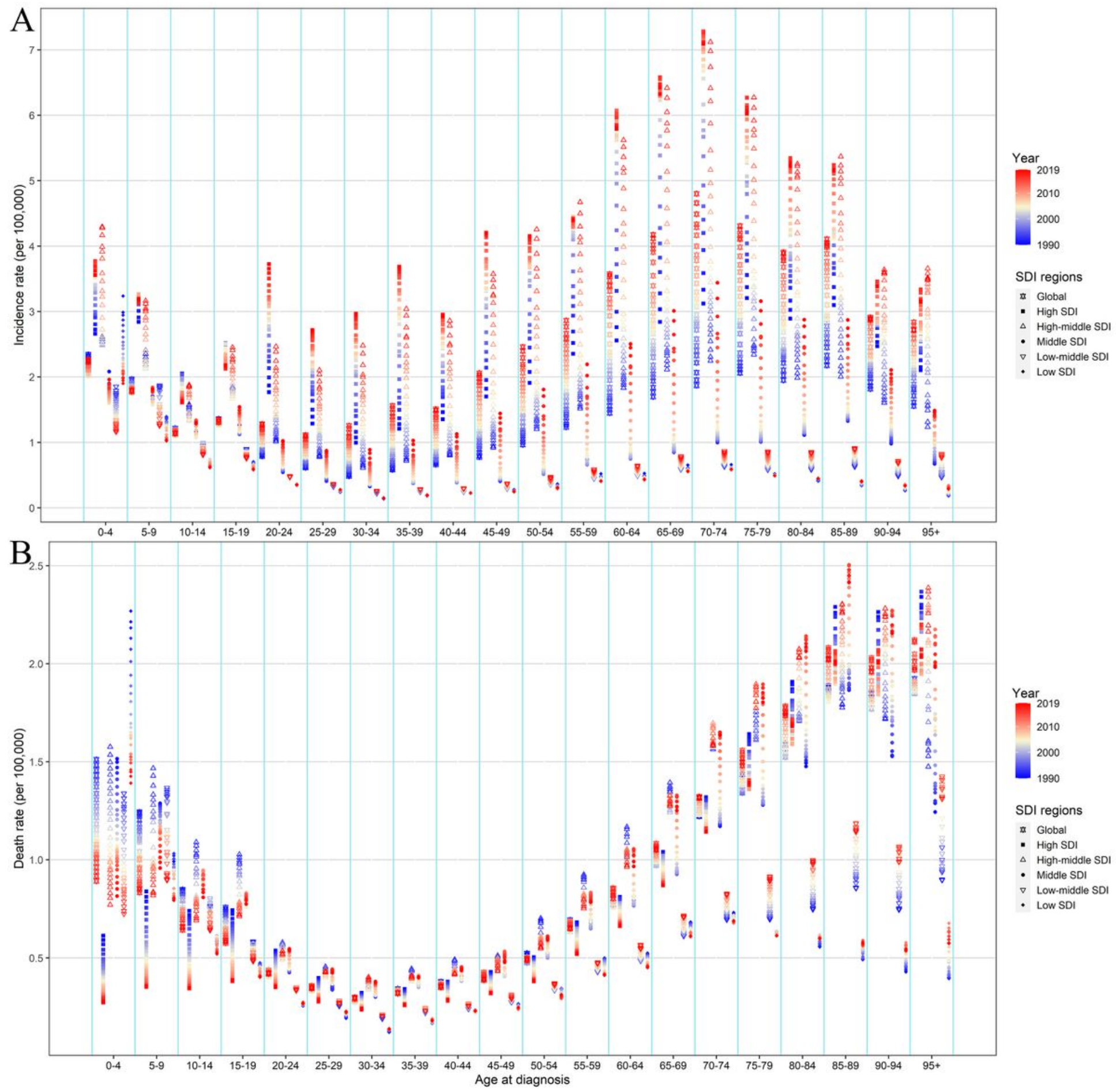

Figure 5

Incidence rate and death rate in different age groups from 1990 to 2019 in globe and SDI regions. A) Incidence rate. B) Death rate. 



Sex/Risk factors

- Male/Smoking

gh body-mass index

- Male/Occupational exposure to benzene

- Femaie/Smokin

- Female/High body-mass index

to benzene

- Female/Occupational exposure to formaldehyde

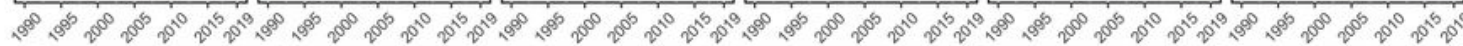
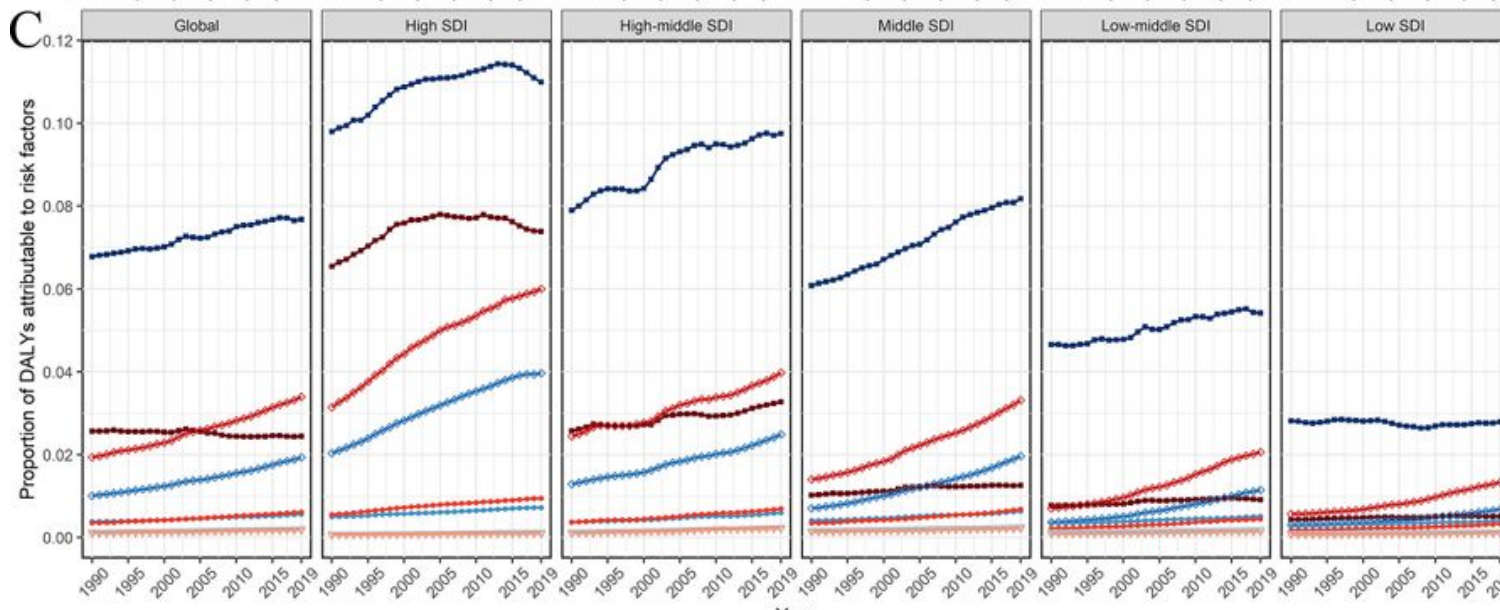

Sex/Risk factors

- Male/Smoking

$\rightarrow$ Male/righ body-mass index

- Ferecupational exposure to formaldehyde

$\rightarrow$ Female/High body-mass index

- Female/Occupational exposure to benzene

- Female/Occupational exposure to formaldehyde

\section{Figure 6}

Risk factors contributing to ALL-related DALYs from 1990 to 2019.

\section{Supplementary Files}

This is a list of supplementary files associated with this preprint. Click to download. 
- Supplementaryinformation1.pdf

- Supplementaryinformation2.pdf 